\title{
Structural Rearrangement of 2-D Zeolite Nanosheets under Electron Beam
}

\author{
Prashant Kumar, ${ }^{1}$ Michael L. Odlyzko, ${ }^{1}$ Neel Rangnekar, ${ }^{1}$ Michael Tsapatsis, ${ }^{1}$ K. Andre Mkhoyan ${ }^{1}$ \\ ${ }^{1}$ Department of Chemical Engineering \& Materials Science, University of Minnesota, Minneapolis, MN 55455.
}

Two-dimensional (2-D) zeolites and zeolite nanosheets are porous silicate frameworks desirable for catalytic uses involving bulky molecules [1], thin film separation membranes [2], and low-k dielectric materials [3]. Functionality of such zeolites is highly dependent on their crystal structure, thickness and pore dimensions. Low-dose transmission electron microscopy (TEM) studies at an optimum accelerating voltage have proven particularly useful in crystallographic structure determination of these electron beam sensitive materials [4]. However, it is known that zeolites undergo amorphization through radiolysis at low accelerating voltages, as well as both sputtering and amorphization through knock-on at high accelerating voltages [5]. Being inherently destructive, the examination of zeolites in TEM cannot perfectly reveal as-synthesized structure.

Here, we investigate the structural evolution of MFI-zeolite nanosheets over time upon electron beam exposure in the TEM. A time series of selected area electron diffraction (SAED) patterns was acquired using a FEI Tecnai G2 F30 (S)TEM with a TWIN pole piece at $300 \mathrm{kV}$ accelerating voltage. Dose was limited to $1.9 \mathrm{e}^{-} / \AA^{2} / \mathrm{s}$ on the specimen.

MFI-nanosheets containing (i) $9 \mathrm{wt} \%$ and (ii) 26-28 wt \% organic structure directing agent (OSDA) were synthesized using a method described by Varoon et al. [6]. The crystal structure of these nanosheets was confirmed to be MFI-zeolite type within 2 minutes of electron beam exposure, using high-resolution bright-field conventional TEM (BF-CTEM) imaging, high angle annular dark field scanning TEM (HAADF-STEM) imaging, and [010] zone axis SAED (Figure 1a-f). The thickness of these nanosheets was determined to be 1.5 unit cells (or $3.2 \mathrm{~nm}$ ) by mapping a rel-rod through a tilt series of SAED patterns (Figure 1g-h). The evolution of diffraction spot intensities under continuous electron beam exposure showed that even though there was evidence of amorphization, some spots increased in intensity over time, confirming that other structural rearrangements occur within these nanosheets (Figure 2a-d).

The observed increases in spot intensity during TEM illumination are explained by the removal of OSDA upon beam exposure: mass loss of OSDA causes the nanosheets to reduce in volume, as well as allows the crystal structure to relax from monoclinic to orthorhombic type. We relate the observed structural changes to the OSDA fraction in these nanosheets by carefully analyzing the diffraction spot intensities and in-plane lattice parameters.

References:

[1] A. Corma et al, Nature 396 (1998), p. 353.

[2] K. Varoon et al, Science 334 (2011), p. 72.

[3] C.M. Lew et al, Acc. Chem. Res. 43 (2010), p. 210.

[4] M.M.J. Treacy, J.M. Newsam, Ultramicroscopy 23 (1987), p. 411.

[5] O. Ugurlu et al, Phys. Rev. B 83 (2011), p. 113408.

[6] K. Varoon et al, AIChE. J. 59 (2013), p. 3458. 
[7] This work was supported as part of the Catalysis Center for Energy Innovation, an Energy Frontier Research Center funded by the U.S. Department of Energy, Office of Science, Basic Energy Sciences under Award DE-SC0001004.
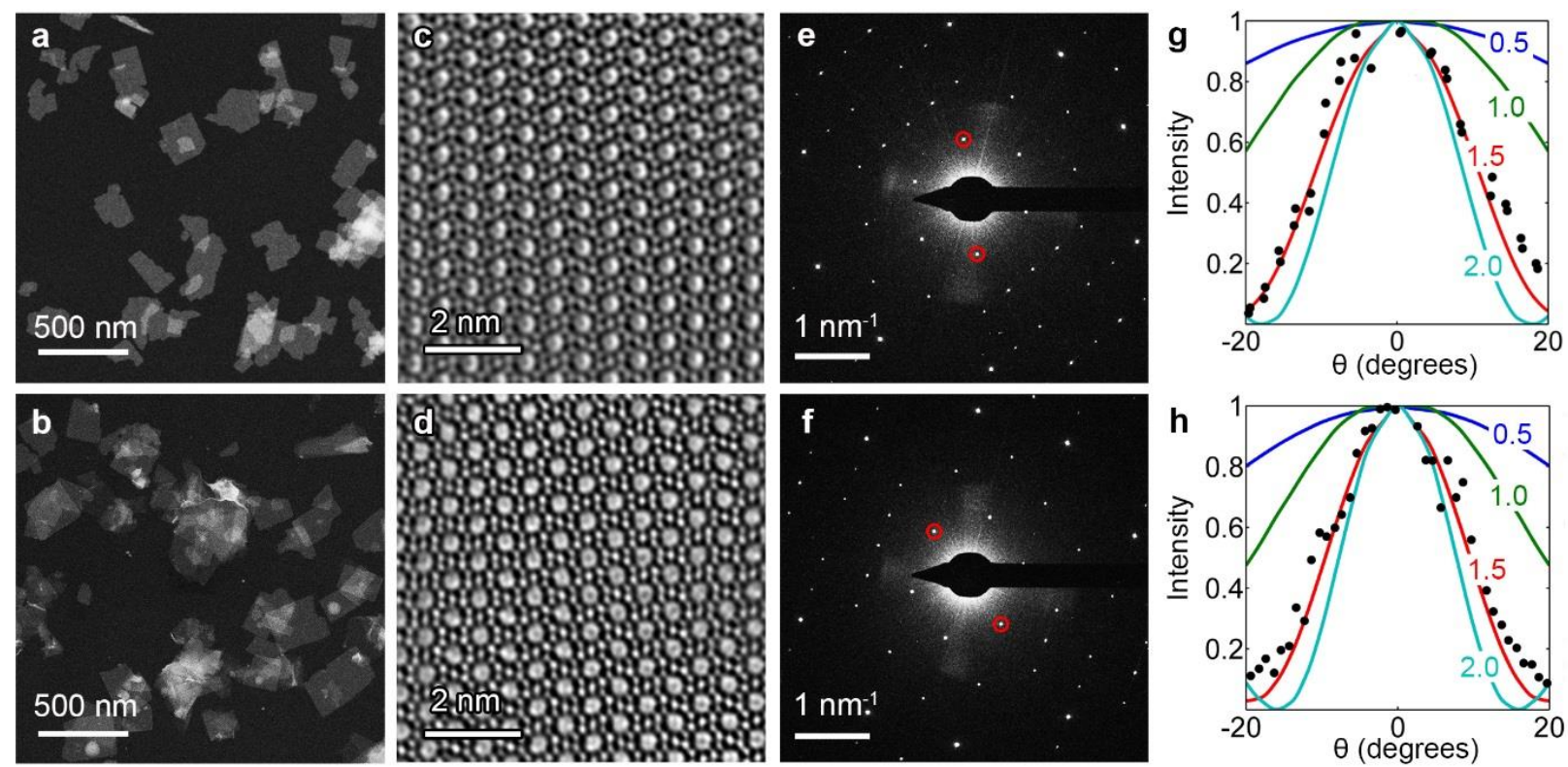

Figure 1. Top and bottom rows correspond to MFI nanosheets containing 9 wt $\%$ OSDA and 27 wt $\%$ OSDA respectively. (a),(b) HAADF-STEM image showing uniformly thick MFI nanosheets. (c),(d) boriented HR-CTEM image confirming MFI structure type. (e),(f) [010] zone axis SAED patterns of MFI nanosheets. (g),(h) Modulation of encircled diffraction spots in (e),(f) with tilt angle. Solid lines represent multislice simulations of spot intensity modulation for $0.5,1.0,1.5$ and 2.0 unit cell thick nanosheets. Black dots represent experimental data.
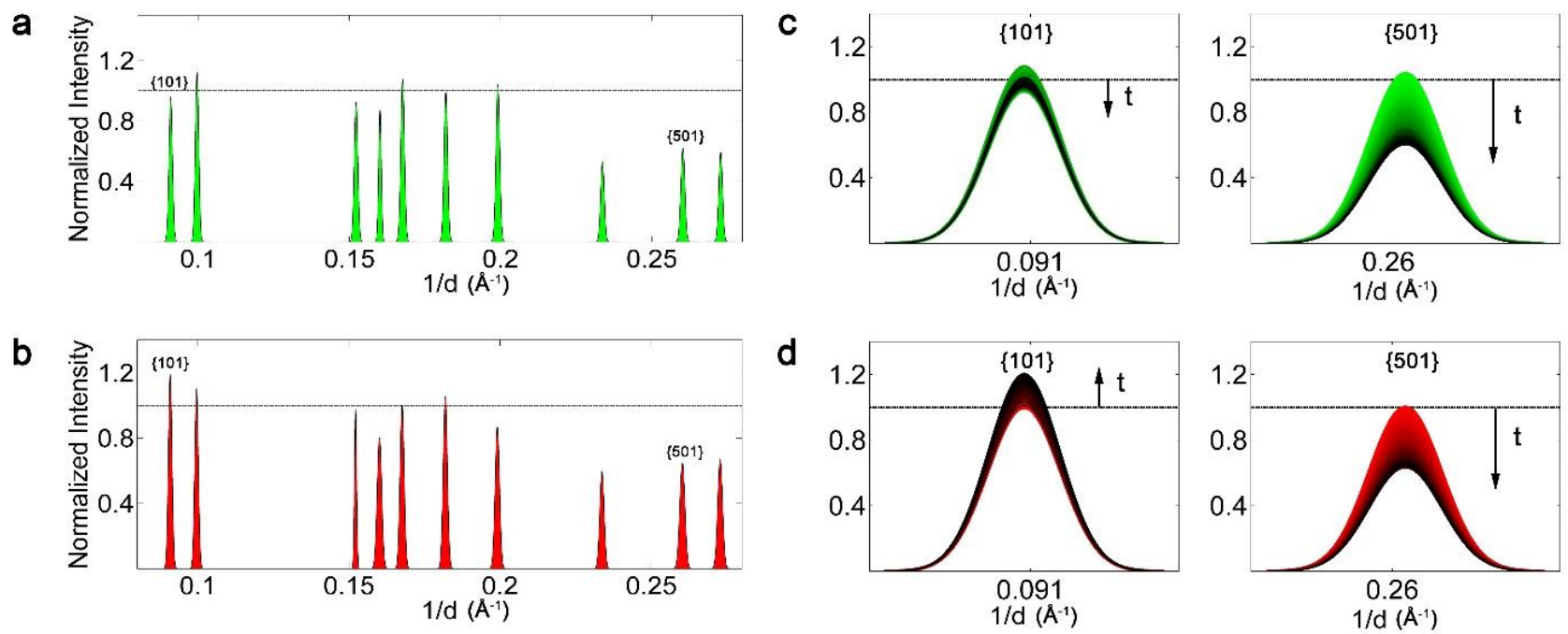

Figure 2. Top and bottom rows correspond to MFI nanosheets containing 9 wt $\%$ OSDA and 27 wt $\%$ OSDA respectively. (a),(b) Normalized SAED peak intensities after 6 hrs of electron beam exposure. Horizontal line represents the spot intensity upon initial exposure to the beam. (c),(d) Evolution of peak intensities shown in (a) and (b) with time. Increasing time from 0-6 hrs is indicated by color gradients fading to black. $\{101\}$ spot intensity increases for nanosheet containing 27 wt\% OSDA while $\{501\}$ spot intensities decay with time for both the samples. 